\title{
Kainic acid induces expression of caveolin-1 in activated microglia in rat brain
}

\author{
Shigeko Takeuchi ${ }^{1}$, Wakoto Matsuda ${ }^{2}$, Ikuo Tooyama ${ }^{1}$, Osamu Yasuhara ${ }^{2,3}$ \\ ${ }^{1}$ Molecular Neuroscience Research Center, Shiga University of Medical Science, Otsu, Japan \\ ${ }^{2}$ Department of Anatomy, Shiga University of Medical Science, Otsu, Japan \\ ${ }^{3}$ University of Shiga Prefecture School of Human Nursing, Hikone, Japan
}

\begin{abstract}
Caveolin-1, a major constituent of caveolae, has been implicated in endocytosis, signal transduction and cholesterol transport in a wide variety of cells. In the present study, the expression of caveolin- 1 was examined by immunohistochemistry in rat brain with or without systemic injection of kainic acid (KA). Caveolin-1 immunoreactivity was observed in capillary walls in brains of control rats. From one to seven days after KA injection, caveolin-1 immunoreactivity appeared in activated microglia in the cerebral cortex, hippocampus and other brain regions. The strongest immunoreactivity of microglia was seen after 3 days after KA administration. The expression of caveolin-1 was confirmed by RT-PCR and Western blot analysis, respectively. The induction of caveolin-1 expression in microglia activated in response to kainic acid administration suggests its possible role in a modulation of inflammation. (Folia Histochemica et Cytobiologica 2013, Vol. 51, No. 1, 25-30)
\end{abstract}

Key words: caveolin 1 expression, brain, microglia, kainic acid, seizures, rat, immunohistochemistry

\section{Introduction}

Caveolin-1 is the major protein component of caveolae that are cholesterol- and sphingolipid-enriched microdomains of the plasma membrane [1]. Because caveolae are believed to be involved in two-way communication and exchange of molecules between the cell and extracellular environment, caveolin-1 has been implicated in multiple cellular functions including endocytosis, signal transduction and cholesterol transport [2]. In addition to caveolae, caveolin-1 was shown to be present in non-caveolar scaffold domains on the plasma membrane [3] and at many other intracellular locations [2]. Probably caveolin-1 plays important multiple biological roles in a heterogeneous cellular microenvironments.

\footnotetext{
Correspondence address: $O$. Yasuhara

University of Shiga Prefecture School of Human Nursing 2500 Hassaka-cho, Hikone 522-8533, Japan;

tel: +81749288645 ;

fax: +8174928 95 13;

e-mail: yasuhara.o@nurse.usp.ac.jp
}

In the brain, caveolin-1 has been reportedly found in endothelial cells and astrocytes [4]. The expression of caveolin-1 was also demonstrated in many types of cells in culture, including astrocytes [5], differentiating PC12 cells, dorsal root ganglion neurons [6], and oligodendrocytes [7]. Furthermore, injuries to the spinal cord caused an increase in phosphorylation of caveolin- 1 in macrophages and microglia $[8,9]$. These results suggest that virtually all types of cells in the nervous system are potentially capable of producing caveolin-1.

In rodents, injection of kainic acid (KA) results in recurrent seizures, behavioral changes, and subsequent degeneration of selective populations of neurons in the brain, showing characteristics similar to human temporal lobe epilepsy [10]. The hippocampus is particularly vulnerable to KA-induced neurotoxicity, where the pathological changes are characterized by pyramidal cell loss associated with glial activation, mossy fiber synaptic reorganization, and increased neurogenesis of dentate granule cells [10-12]. Thus, KA administration in rodents has widely been used as a model to elucidate the mechanisms of neurotoxicity of excitatory neurotransmitters. Bu et al. [13] 
demonstrated that KA and other glutamate receptor agonists induce expression of caveolin-1 in a concentration-dependent manner in cultured primary hippocampal neurons of rats. This observation strongly suggests a role of caveolin-1 in KA-induced neuronal damage. To clarify the involvement of caveolin- 1 in KA-induced excitotoxicity, we examined in the present study expression of caveolin-1 and its mRNA in rat brain after KA administration, by RT-PCR, Western blot analysis and immunohistochemistry.

\section{Material and methods}

Animals. Male Wistar rats (Clea Japan Inc., Tokyo, Japan), weighing $250-300 \mathrm{~g}$, were used. The rats were handled in compliance with the principles of the National Institute of Health Guide for the Care and Use of Laboratory Animals (NIH Publications No. 80-23, revised 1986). All efforts were made to minimize both the number of animals used and any suffering that they might experience. The rats received a single intraperitoneal (i.p.) injection of KA (Sigma, St. Louis, MO, USA; $12 \mathrm{mg} / \mathrm{kg}$ dissolved in physiological saline). Only the rats which showed generalized seizures (tonic-clonic convulsions) were used in this study.

Immunohistochemistry. Caveolin-1 immunohistochemistry was performed using the rats with survival periods of one, three and seven days after KA injection $(n=3$ in each group). The rats injected with physiological saline served as the control group $(1,3$, and 7 days post-injection; $\mathrm{n}=2$ in each group). Each animal was perfused through the ascending aorta with $10 \mathrm{mM}$ phosphate-buffered saline (PBS, $\mathrm{pH}$ 7.4), followed by a fixative of $4 \%$ paraformaldehyde in 0.1 $\mathrm{M}$ phosphate buffer ( $\mathrm{PB}, \mathrm{pH}$ 7.4). The brains were removed from the skull and postfixed overnight in a fixative of $4 \%$ paraformaldehyde in $0.1 \mathrm{M} \mathrm{PB}$ at $4^{\circ} \mathrm{C}$. After cryoprotection with $0.1 \mathrm{M}$ PB containing $15 \%$ sucrose, $20 \mu \mathrm{m}$-thick sections were cut in a cryostat and stored in 0.1 M PBS containing $0.3 \%$ Triton X-100 (PBST) until used.

The sections, in a free-floating state, were incubated overnight with a rabbit polyclonal antibody against caveolin-1 (N-20; Santa Cruz Biotechnology, Santa Cruz, CA, USA; diluted $1: 4000)$ at $4^{\circ} \mathrm{C}$, for $2 \mathrm{~h}$ with biotinylated antirabbit IgG (Vector Labs, Burlingame, CA, USA; diluted 1: $1000)$ at room temperature, and for $1 \mathrm{~h}$ with the avidinbiotinylated peroxidase complex (ABC Elite; Vector; diluted 1:2000) at room temperature. Dilution of the reagents and washing of sections between each step were done with PBST. Color was developed by reacting the sections for 10 min with a mixture containing $0.02 \% 3,3$ '-diaminobenzidine, $0.0045 \% \mathrm{H}_{2} \mathrm{O}_{2}$ and $0.3 \%$ nickel ammonium sulfate in $50 \mathrm{mM}$ Tris- $\mathrm{HCl}$ buffer ( $\mathrm{pH}$ 7.6). The stained sections were airdried, washed in tap water, dried through a graded series of alcohol, cleared with xylene, and coverslipped with Entel- lan (Merck, Darmstadt, Germany). For immunohistochemical controls, the caveolin-1 antibody which had been preincubated overnight with the blocking peptide of caveolin1 (sc-894P, Santa Cruz Biotechnology, Santa Cruz, CA, USA) was applied to immunohistochemical procedures. No positive staining was observed in these control studies.

To characterize caveolin-1-positive cells, sections from a rat 3 days after KA injection were doubly stained for caveolin-1 as described above, and one of the following three marker proteins. They were NeuN for neurons (MAB377, mouse monoclonal; Chemicon, Temecula, CA, USA; diluted 1:1000), glial fibrillary acidic protein (GFAP) for astrocytes (GF12.24, mouse monoclonal; Progen, Heidelberg, Germany; 1:10), and CD11b for activated microglia (OX42, mouse monoclonal; Serotec, Raleigh, NC, USA; diluted 1:500). After overnight-incubation in a mixture of primary antibodies, the sections were covered for $2 \mathrm{~h}$ with a mixture of Alexa Fluor 488-conjugated anti-rabbit IgG (for caveolin-1) and Alexa Fluor 594-conjugated anti-mouse IgG (for NeuN, GFAP or CD11b) (all made in goat; Molecular Probes Inc., Eugene, OR, USA; 1:1000) at room temperature. After washing, the mounted sections were analyzed with a Zeiss LSM 510 confocal laser scanning microscope (Carl Zeiss, Thornwood, NY) equipped with an argon laser $(458 / 488 / 514 \mathrm{~nm})$, and a green helium/neon laser (543 nm). Single optical slice images were taken using $40 \times$ LD Achroplan air interface objective lens at a z-axis step of $0.6 \mu \mathrm{m}$ depth.

RT-PCR. After perfusion with $10 \mathrm{mM}$ PBS, brains were removed from a saline-injected control rat and a KA-injected rat 3 days post-injection. Total RNA was extracted from fresh tissues of right cerebral cortex and hippocampus using the acid guanidium thiocyanate-phenol method. Five $\mu \mathrm{g}$ of each total RNA was reversely transcribed for the synthesis of the first strand cDNA using 500 pmol of oligo$\mathrm{dT}_{12-18}$ (Amersham Biosciences Corp., Arlington Heights, IL) and 200 units of SuperScript II (Gibco BRL, Gaithersburg, MD).

PCR was performed in the GeneAmp PCR System 2400 (Perkin Elmer, Foster City, CA) using a primer pair specific to rat caveolin-1 cDNA. The exact nucleotide sequences of the primers were; 5'-CTACAAGCCCAACAACAAGGC-3' (5'-sense primer), and 5'-AGGAAGCTCTTGATGCACGGT-3' (3'-antisense primer) (GenBank no. AF439778). For a control, PCR for $\beta$-actin gene was done in a separate tube. The sequences of the primers for rat $\beta$-actin were: 5 '-TGGTGGGTATGGGTCAGAAGGACTC-3' (5'-sense primer), and 5'-CATGGCTGGGGTGTTGAAGGTCTCA-3' ( 3 '-antisense primer). The expected sizes of the PCR products were 342 base pair (bp) for caveolin-1 and $265 \mathrm{bp}$ for $\beta$-actin, respectively.

Each $25 \mu \mathrm{l}$ reaction mixture consisted of $2 \mathrm{ng} / \mu \mathrm{l}$ of the template cDNA, $0.8 \mu \mathrm{M}$ of each of the primers, $0.2 \mathrm{mM}$ of 

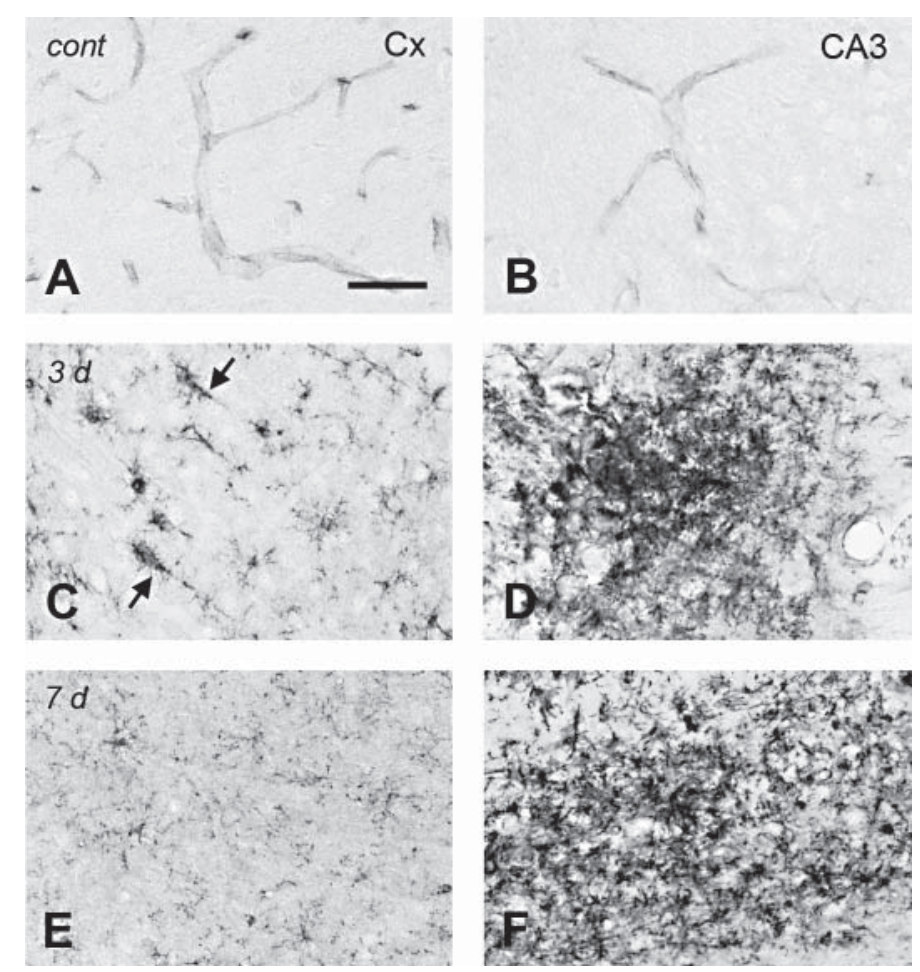

Figure 1. Immunostaining for caveolin-1 in the parietal cortex $(\mathbf{A}, \mathbf{C}, \mathbf{E})$ and the CA3 area of the hippocampus $(\mathbf{B}, \mathbf{D}, \mathbf{F})$ from control and KA-treated rats. (A, B) A saline-injected control rat, showing positive capillary staining. (C, D) A rat 3 days after KA injection. Intensely stained cells appear in the cortex and are accumulated in the CA3. Arrows in C show positive cells with pyramidal morphology. (E, F) A rat 7 days after KA injection. Weakly stained positive cells are present in the cortex, but intensely stained cells are aggregated in the CA3. Bar $=50 \mathrm{~mm}$

each of 4 deoxynucleotide triphosphates and 0.25 units of AmpliTaq Gold polymerase (Perkin Elmer) in 1xPCR buffer (Perkin Elmer). After preincubation for $10 \mathrm{~min}$ at $95^{\circ} \mathrm{C}$, the amplification program was performed for 34 cycles with denaturation for $0.5 \mathrm{~min}$ at $95^{\circ} \mathrm{C}$, annealing for $0.5 \mathrm{~min}$ at $64^{\circ} \mathrm{C}$, and extension for $1.5 \mathrm{~min}$ at $72^{\circ} \mathrm{C}$. The PCR products were electrophoresed on a $3 \%$ agarose gel and stained with ethidium bromide. After dissecting out the expected bands from the gel, the target PCR product was eluted, and DNA was cloned using a TA cloning system (Invitrogen Corp., Carlsbad, CA), followed by sequencing using the ABI-PRISM cycle sequencing kit and 310 DNA sequencer (Perkin Elmer).

Western blot analysis. After perfusion with $10 \mathrm{mM}$ PBS, tissues of right cerebral cortex and hippocampus were obtained from both a saline-injected control rat and a rat 3 days after KA injection. They were homogenized in 10 volumes of ice-cold $20 \mathrm{mM}$ Tris- $\mathrm{HCl}(\mathrm{pH} 7.5), 0.15 \mathrm{M} \mathrm{NaCl}$, $1 \%$ Triton $\mathrm{X}-100$, and $60 \mathrm{mM}$ octyl-glucoside, under the presence of protease inhibitor cocktail tablets, Complete Mini (Roche Diagnostics, Mannheim, Germany; one tablet/10 mL). The homogenates were centrifuged at $14000 \mathrm{~g}$ for $20 \mathrm{~min}$ at $4^{\circ} \mathrm{C}$. About $25 \mu \mathrm{g}$ of the crude extracted protein and Prestained Precision Protein Standards (Bio-Rad,
Hercules, CA, USA) were electrophoresed on a $10 \%$ sodium dodecyl sulfate (SDS)-polyacrylamide gel under a reducing condition, and then transferred to a polyvinylidene difluoride membrane (Immobilon-P; Millipore, Tokyo, Japan). The membrane was incubated overnight with the rabbit antibody against caveolin-1 (N20; Santa Cruz Biotechnology; diluted $1: 2000$ ) in $25 \mathrm{mM}$ TBS containing $0.1 \%$ Tween 20 (TBST) at $4^{\circ} \mathrm{C}$, and then for $2 \mathrm{~h}$ with a peroxidase-coupled anti-rabbit IgG Fab' fragment (Histofine; Nichirei Corporation, Tokyo, Japan; diluted 1:100). The peroxidase labeling was detected by chemiluminescence using the SuperSignal West Pico Chemiluminescent Substrate (Pierce, Rockford, IL, USA).

\section{Results}

In saline-injected control rats, capillary walls were positively stained for caveolin-1 in many brain regions including the cerebral cortex (Figure 1A) and hippocampus (Figure 1B). One day after KA administration, caveolin-1-positive cells appeared in the brain including the cerebral cortex and hippocampus. Three days after KA injection, the number of positive cells increased in the cerebral cortex (Figure 1C). Many 

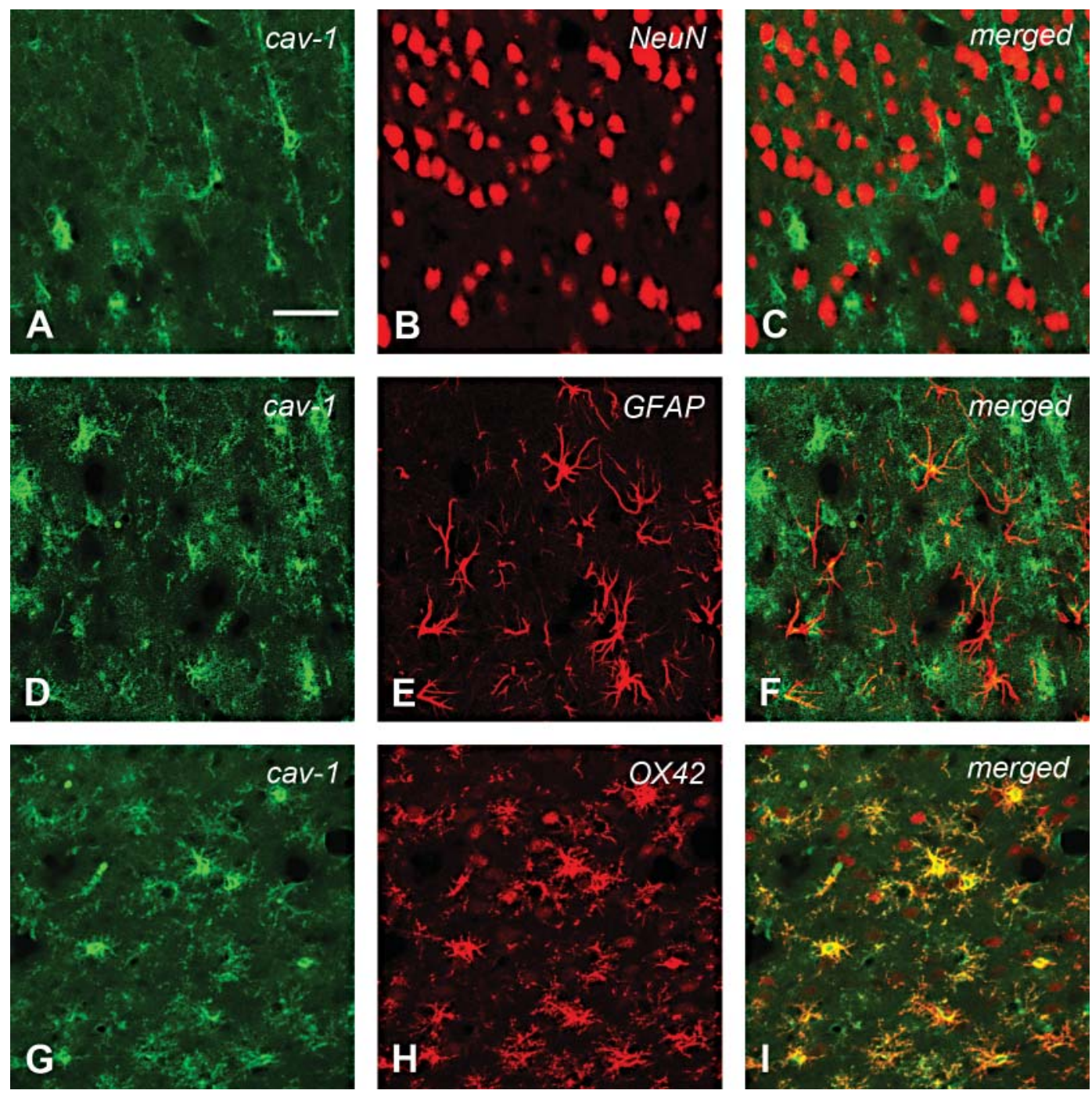

Figure 2. Confocal fluorescent images of the cerebral cortex doubly stained for caveolin-1 and one of the cell marker antigens. (A-C) Caveolin-1-positive cells (green; A), NeuN-positive neurons (red; B), and a composite image of A and B (C), showing no overlap of these two types of cell. (D-F) Caveolin-1-positive cells (green; D), GFAP-positive astrocytes (red; E), and a composite image of D and E (F), showing only a partial overlap of these two types of cell. (G-I) Caveolin1-positive cells (green; G), OX42-labelled microglia (red; H), and a composite image of G and H (I), showing an overlap of caveolin-1-positive cells and OX42-labelled microglia (yellow). Bar $=50 \mathrm{~mm}$

immunoreactive (ir) positive cells had small, round to ovoid cell bodies with several processes, while some positive cells showed pyramidal profiles (Figure 1C). In the hippocampus, particularly in the CA3 area, a dense accumulation of ir-positive cells was observed (Figure 1D). Similar aggregations of immunoreactive cells were also detected in the thalamus and entorhinal cortex.

To better characterize ir-positive cells, brain sections of the rats 3 days after KA injection were doubly stained for caveolin- 1 and one of the selected three markers of various brain cell populations. As shown in Figure 2, caveolin-1 immunoreactivity was observed in virtually all CD11b-positive (OX42-labelled) mi- croglia and only a few GFAP-positive astrocytes, but not in NeuN-positive neurons. Thus, the major population of caveolin-1-positive cells was found to consist of activated microglia.

Seven days after KA-injection, the number and staining intensity of positive microglia decreased in the cerebral cortex (Figure 1E). Only a few positive cells showed morphology of activated microglia. Positive microglial aggregates were found in the regions vulnerable to the toxic effects of KA, such as the CA3 area of the hippocampus (Figure $1 \mathrm{~F}$ ), some thalamic nuclei, amygdala and entorhinal cortex.

Figure 3A shows expression of caveolin-1 mRNA analyzed by RT-PCR. As shown, the amplification 
A Caveolin-1

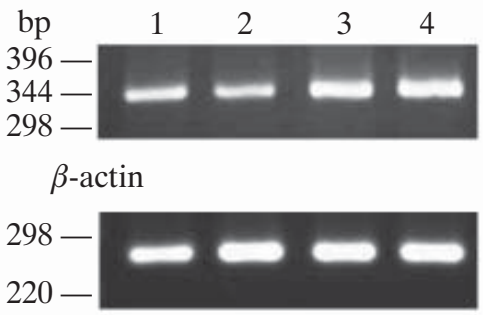

B

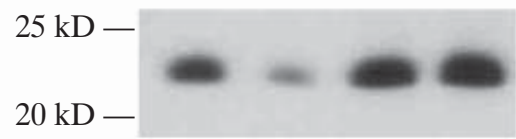

Figure 3. RT-PCR and Western blot analysis for caveolin-1. Lanes 1-4: right cerebral cortex (lane 1) and right hippocampus (lane 2) from a saline-injected rat, and right cerebral cortex (lane 3 ) and right hippocampus (lane 4) from a rat 3 days after KA injection. (A) RT-PCR for caveolin- 1 and $\beta$-actin mRNA. The products with the expected sizes (342 bp for caveolin-1, and $265 \mathrm{bp}$ for $\beta$-actin, respectively) are detectable. The locations of the molecular size markers are indicated on the left. (B) Western blot analysis, showing the positive bands at about $22 \mathrm{kDa}$ in all samples. The molecular weights of the size markers are indicated on the left

product for the caveolin-1 gene was detected with the expected size ( $342 \mathrm{bp}$ ) in the cerebral cortex and hippocampus of both saline-injected and KA-treated rats. The nucleotide sequence analysis confirmed the identity of the PCR product with the caveolin-1 gene fragment. No mutation was found within the nucleotide sequence of analyzed fragments. In Western blot analysis, the caveolin- 1 antibody revealed a positive band of about $22 \mathrm{kDa}$ in the cerebral cortex and hippocampus of both saline-injected and KA-treated rats (Figure 3B).

\section{Discussion}

In the present study, two major types of cells are recognized to express caveolin-1 in rat brain. First, caveolin-1 immunoreactivity was present in capillary endothelial cells in vehicle-injected control rats. Second, caveolin-1-positive microglia were widely distributed in the brain areas including the cerebral cortex and hippocampus from 1 day to 3 days after injection. Seven days post-injection, positive microglia were confined to such vulnerable areas as the hippocampus, thalamus and entorhinal cortex. The present study demonstrates for the first time that the expression of caveolin-1 is induced in activated microglia in rat brain after systemic treatment with KA. The ex- pression of caveolin- 1 and its mRNA in the brain of KA-treated rats were confirmed by Western blot analysis and RT-PCR, respectively, though the changes in their expression levels should be determined by future quantitative analyses.

Microglia and macrophages are the cells of the mononuclear phagocyte lineage originating from monocytes. Although the expression of caveolin-1 in immune cells has long been debated [14], a body of evidence indicates that macrophages actually express caveolin-1 in pathological conditions [15]. In addition, it is reported that the expression of caveolin- 1 gene is up-regulated in monocytes after activation by adhesion to endothelial cells [16]. The present study provides additional evidence indicating that caveolin-1 plays roles in tissue injuries through cells of the mononuclear phagocytic system. Because CD11b antigen is expressed by two groups of morphologically indistinguishable phagocytic cells, resident brain microglia and infiltrating peripheral macrophages [17], the exact origin of the caveolin-1-positive brain cells remains to be determined. Although a previous in vitro study has shown that cultured primary hippocampal neurons from rats express caveolin-1 after excitotoxic treatment [13], the present study failed to show any caveolin-1-positive neurons in KA-treated rat brains.

The precise functional role of caveolin- 1 in activated microglia is unclear. It is probable that caveolin-1 is involved in lipid metabolism in microglia [14]. More importantly, it is suggested that caveolin- 1 is a potent modulator of inflammation. Bucci et al. [18] showed a role of caveolin-1 in suppression of inflammation, by using the scaffoling domain of caveolin-1 to inhibit edema formation and vascular leakage in rats. Such anti-inflammatory effects of caveolin-1 have partly been ascribed more directly to the action of caveolin-1 in macrophages. For example, caveolin-1 deficient macrophages display impaired phagocytosis [19]. Wang et al. [20] demonstrated that caveolin1 acts as a potent immunomodulatory effecter molecule in murine alveolar and peritoneal macrophages. They showed that caveolin-1 has a protective role for inflammation by suppression of pro-inflammatory cytokine production (TNF- $\alpha$ and IL-6) and augmentation of anti-inflammatory cytokine production (IL-10) [20]. Thus, it is tempting to speculate that caveolin-1 in activated microglia participates in modulation of inflammation in the brain in response to excitotoxic insults.

In summary, we have demonstrated for the first time that KA administration induces the expression of caveolin-1 in activated microglia in rat brain. The results suggest an immunomodulatory role of caveolin-1 in KA-induced brain damage. Because activat- 
ed microglia are known to act both neurotoxic and neuroprotective effects [21], it is unclear whether the action of microglial caveolin-1 is toxic or protective to neurons. Another question is whether caveolin-1 induced in activated microglia by KA is phosphorylated or not, because an increase in phosphorylation of caveolin- 1 has been reported in microglia in rat spinal cord in some experimental conditions [8, 9, 22]. In addition, the apparent alterations in the expression level of caveolin-1 should be clarified by further quantitative analysis. Future studies are needed to address these issues.

\section{Acknowledgments}

We thank Mr. M. Suzaki and T. Yamamoto (The Central Laboratory, Shiga University of Medical Science), and Ms. A. Yamaguchi (Department of Anatomy, Shiga University of Medical Science) for technical assistance.

\section{References}

1. Engelman JA, Zhang X, Galbiati F et al. Protein comlexes 98'. Molecular genetics of the caveolin gene family: Implications for human cancers, diabetes, Alzheimer disease, and muscular dystrophy. Am J Hum Genet. 1998; 63: 1578-1587.

2. Quest AFG, Leyton L, Parraga M. Caveolins, caveolae, and lipid rafts in cellular transport, signaling, and disease. Biochem Cell Biol. 2004;82:129-144.

3. Lajoire P, Goetz JG, Dennis JW, Nabi IR. Lattices, rafts, and scaffolds: domain regulation of receptor signaling at the plasma membrane. J Cell Biol. 2009;185:381-385.

4. Ikezu T, Ueda H, Trapp BD et al. Affinity-purification and characterization of caveolins from the brain: Differential expression of caveolin-1, -2, and -3 in brain endothelial and astroglial cell types. Brain Res. 1998; 804: 177-192.

5. Cameron PL, Ruffin JW, Bollag R, Rasmussen H, Cameron RS. Identification of caveolin and caveolin-related proteins in the brain. J Neurosci. 1997;17:9520-9535.

6. Galbiati F, Volonté D, Gil O et al. Expression of caveolin-1 and -2 in differentiating PC12 cells and dorsal root ganglion neurons: Caveolin-2 is up-regulated in response to cell injury. Proc Natl Acad Sci USA. 1998;95:10257-10262.

7. Arvanitis DN, Wang H, Bagshaw RD, Callahan JW, Boggs JM. Membrane-associated estrogen receptor and caveolin-1 are present in central nervous system myelin and oligodendrocyte plasma membranes. J Neurosci Res. 2004;75:603-613.

8. Kim H, Moon C, Kim J et al. Increased phosphorylation of caveolin-1 in the spinal cord of irradiated rats. J Vet Sci. 2007;8:323-327.

9. Shin T. Increases in the phosphorylated form of caveolin-1 in the spinal cord of rats with clip compression injury. Brain Res. 2007;1141:228-234.

10. Lothman EW, Collins RC. Kainic acid induced limbic seizures: metabolic, behavioral, electroencephalographic and neuropathological correlates. Brain Res. 1981;218:299-318.

11. Nakagawa E, Aimi Y, Yasuhara O, Tooyama I, Shimada M, McGeer PL, Kimura H. Enhancement of progenitor cell division in the dentate gyrus triggered by initial limbic seizures in rat models of epilepsy. Epilepsia 2000;41:10-18.

12. Zhang X-M, Zhu J. Kainic acid-induced neurotoxicity: targeting glial responses and glia-derived cytokines. Curr Neuropharmacol. 2011;9:388-398.

13. Bu J, Bruckner SR, Sengoku T, Geddes JW. Glutamate regulates caveolin expression in rat hippocampal neurons. J Neurosci Res. 2003;72:185-190.

14. Gargalovic P, Dory L. Caveolins and macrophage lipid metabolism. J Lipid Res. 2003;44:11-21.

15. Harris J, Werling D, Hope JC, Taylor G, Howard CJ. Caveolae and caveolin in immune cells: distribution and functions. Trends Immunol. 2002;23:158-164.

16. Thomas-Ecker S, Lindecke A, Hatzmann W, Kaltschmidt C, Zänker KS, Dittmar T. Alteration in gene expression pattern of primary monocytes after adhesion to endothelial cell. Proc Natl Acad Sci USA. 2007;104:5539-5544.

17. Ji KA, Yang MS, Jeong HK, Mink KJ, Kang SH, Joe EH. Resident microglia die and infiltrated neutrophils and monocytes become major inflammatory cells in lipopolysaccharideinjured brain. Glia 2007;55:1577-1588.

18. Bucci M, Gratton JP, Rudic RD et al. In vivo delivery of the caveolin-1 scaffolding domain inhibits nitric oxide synthesis and reduces inflammation. Nat Med. 2000;6:1362-1367.

19. Li J, Scherl A, Medina F et al. Impaired phagocytosis in caveolin-1 deficient macrophages. Cell Cycle 2005;4:1599-1607.

20. Wang XM, Kim HP, Song R, Choi AMK. Caveolin-1 confers anti-inflammatory effects in murine macrophages via the MKK3/p38 MAPK pathway. Am J Respir Cell Mol Biol. 2006; $34: 434-442$

21. Minghetti L, Levi G. Microglia as effector cells in brain damage and repair: focus on prostanoids and nitric oxide. Prog Neurobiol. 1998;54:99-125.

22. Kim H, Ahn M, Lee J et al. Increased phosphorylation of caveolin-1 in the spinal cord of Lewis rats with experimental autoimmune encephalomyelitis. Neurosci Lett. 2006;402:76-80.

Submitted: 18 October, 2012

Accepted after reviews: 6 March, 2013 